\title{
Generation of Large Moments in a Spin-1 Chain with Random Antiferromagnetic Couplings
}

\author{
Kun Yang ${ }^{1,2}$ and R. N. Bhatt ${ }^{1}$ \\ ${ }^{1}$ Department of Electrical Engineering, Princeton University, Princeton, New Jersey 08544 \\ ${ }^{2}$ Condensed Matter Physics 114-36, California Institute of Technology, Pasadena, California 91125
}

(Received 22 December 1997)

\begin{abstract}
We study the spin-1 chain with nearest neighbor couplings that are rotationally invariant, but include both Heisenberg and biquadratic exchange, with random strengths. We demonstrate, using perturbative renormalization group methods as well as exact diagonalization of clusters, that the system generates ferromagnetic couplings under certain circumstances even when all the bare couplings are antiferromagnetic. This disorder induced instability leads to formation of large magnetic moments at low temperatures, and is a purely quantum mechanical effect that does not have a classical counterpart. The physical origin of this instability, as well as its consequences, are discussed. [S0031-9007(98)06158-4]
\end{abstract}

PACS numbers: 75.10.Jm, 75.30.Hx, 75.50.Ee

Classical spin models have been studied extensively over the past half century. The inclusion of quantum mechanical nature of the spin variables results usually in quantitative effects such as renormalization of transition temperatures, order parameter in the ordered phase, spin wave velocity, etc. However, it began to be realized about two decades back that quantum fluctuations could result in qualitative changes such as the nature of the phase itself. Ma et al. [1] showed that in a spin-1/2 chain with nearest neighbor antiferromagnetic Heisenberg interactions of random strength, quantum fluctuations lead to a divergent density of excitations at low energy scales even in the absence of such divergence in the distribution of bare couplings. Around the same time, Bhatt and Lee [2] independently showed that this occurs for highly disordered spin-1/2 antiferromagnets with short range interactions in higher dimensions as well, and that this phenomenon is a purely quantum mechanical effect [3]. A few years later, Haldane [4] showed that the uniform integer spin chains with antiferromagnetic Heisenberg interactions exhibited a gap, in contrast with the half integer or classical spin chains. The Haldane gap, which scales as $\exp (-\pi S)$ with the spin $S$, is also a purely quantum mechanical effect, unobtainable from the classical $(S \rightarrow \infty)$ limit, where it appears as an essential singularity. Recently, studies of the quantum Ising model in a transverse field in one, two, and three dimensions [5,6] as well as Heisenberg spin chains [7-9] with randomness, have demonstrated that the ground state $(T=0)$ phase diagram includes a Griffiths phase with divergent response functions due to rare fluctuations. Thus, electronic systems in the quantum regime display a richer variety of phenomena resulting from the interplay between correlation and disorder, than is usual for classical systems.

In this paper, we describe another phenomenon occurring in spin chains as a purely quantum mechanical effect-namely, the generation of ferromagnetic $(\mathrm{F})$ couplings, and consequently large moments leading to a Curie susceptibility at low temperatures, in a spin-1 chain with isotropic but random antiferromagnetic (AF) couplings. Though the generated moments become arbitrarily large in the low temperature limit, the phenomenon of the generation of ferromagnetic couplings relies on a purely quantum mechanical effect, and has no classical analog.

For the random spin- $1 / 2$ chain with near neighbor AF interactions, Fisher [10] showed that the real space renormalization group (RG) scheme [1,2] becomes asymptotically exact, and leads to a "random singlet" phase, where distant pairs of spins form singlets in a hierarchical manner dependent on the realization of the random bonds, and dominate the low energy physics. In the presence of randomly placed ferromagnetic couplings of arbitrary concentration, however, Westerberg et al. [11] showed that the random singlet phase is destroyed in one dimension due to the formation of large moments by active, ferromagnetically coupled spins, and the magnetic susceptibility at asymptotically low temperature assumes a pure Curie $(1 / T)$ form, right up to the ferromagnetic point at zero concentration of antiferromagnetic bonds.

For a spin- $1 / 2$ system, spin rotational symmetry, when present, uniquely constrains the coupling between spins to be the Heisenberg form, $J \mathbf{S}_{i} \cdot \mathbf{S}_{j}$. For spins with $S>$ $1 / 2$, however, the most general form of isotropic coupling between spins $i$ and $j$ is $H_{i j}=\sum_{n=1}^{2 S} J^{(n)}\left(\mathbf{S}_{i} \cdot \mathbf{S}_{j}\right)^{n}$. For the random spin-1 chain with nearest neighbor couplings, this implies a Hamiltonian written most generally as

$$
\begin{aligned}
H=\sum_{i}\left[J_{i} \mathbf{S}_{i} \cdot \mathbf{S}_{i+1}\right. & \left.+D_{i}\left(\mathbf{S}_{i} \cdot \mathbf{S}_{i+1}\right)^{2}\right] \\
=\sum_{i} \sqrt{J_{i}^{2}+D_{i}^{2}} & {\left[\cos \theta_{i} \mathbf{S}_{i} \cdot \mathbf{S}_{i+1}\right.} \\
& \left.+\sin \theta_{i}\left(\mathbf{S}_{i} \cdot \mathbf{S}_{i+1}\right)^{2}\right],
\end{aligned}
$$

where the $J$ 's and $D$ 's are uncorrelated random variables.

With purely Heisenberg couplings, it was shown [8] that as long as there are no F bonds in the bare Hamiltonian, the system cannot be in the pure Curie paramagnetic phase. 
In this paper we show that this is no longer the case when biquadratic couplings are present [12].

With no randomness, the properties of the Hamiltonian (2) are controlled by the angular variable $\theta$ satisfying $\tan \theta=D / J$. There exist four different phases (see Fig. 1). For $\pi / 2<\theta<5 \pi / 4$, each individual bond favors a total spin $S_{\text {tot }}=2$ state for the pair it connects, and the ground state of the entire chain is the spin fully polarized ferromagnetic state. For $-\pi / 4<\theta<$ $\pi / 4$ the system is in the Haldane gapped phase [4]. For $-3 \pi / 4<\theta<-\pi / 4$ the chain is spontaneously dimerized [13], while an extended gapless phase has been predicted in the region $\pi / 4<\theta<\pi / 2$ [14]. Except for the ferromagnetic phase, the other phases all have singlet ground states with unbroken spin-rotational symmetry, and we therefore refer to bonds in this region as antiferromagnetic.

In the presence of strong randomness, the system may be studied using a hierarchical real space RG approach. We search for the bond in the system with the largest gap separating its ground state and lowest energy excited state, say the bond coupling spins 2 and 3, with coupling constants $J_{2}$ and $D_{2}$ [see Fig. 2(a)]. If the ground state of this bond is a singlet $\left(-3 \pi / 4<\theta_{2}<\arctan \frac{1}{3}\right)$, then spins 2 and 3 form an inert singlet in the low-energy states of the system, and mediate effective couplings between their neighboring spins 1 and 4, which may be calculated using second order perturbation theory [15]:

$$
\begin{gathered}
\tilde{J}_{14}=\frac{\left(2 J_{1}-D_{1}\right)\left(2 J_{3}-D_{3}\right)}{3\left(J_{2}-3 D_{2}\right)}-\frac{D_{1} D_{3}}{9\left(J_{2}-D_{2}\right)} ; \\
\tilde{D}_{14}=-\frac{2 D_{1} D_{3}}{9\left(J_{2}-D_{2}\right)} .
\end{gathered}
$$

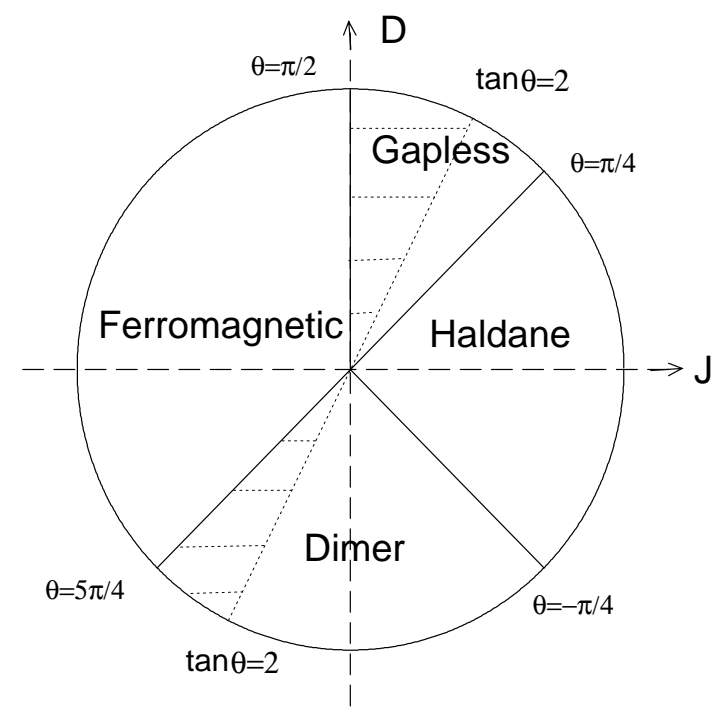

FIG. 1. Phase diagram of a pure spin-1 chain. Solid lines are phase boundaries. Hatched regions represent couplings in the antiferromagnetic sector satisfying $J-D / 2<0$, which, in the presence of randomness, could generate effective ferromagnetic bonds at low energies.
If the ground state of the bond is a triplet $\left(\arctan \frac{1}{3}<\right.$ $\theta_{2}<\pi / 2$ ), spins 2 and 3 form an effective spin $2^{\prime}$ with $S_{2^{\prime}}=1$, and its couplings to its neighbor spin 1 are

$$
\begin{gathered}
\tilde{J}_{12^{\prime}}=\left(J_{1}-D_{1}\right) / 2 ; \\
\tilde{D}_{12^{\prime}}=-D_{1} / 2 .
\end{gathered}
$$

Couplings to spin 4 have identical expressions. Should the ground state of the bond be a quintuplet, an effective spin with $S=2$ forms, and the structure of the original spin-1 chain gets distorted.

Examination of Eqs. (2) and (3) shows that the generated bond may be ferromagnetic, even if all the bonds involved are antiferromagnetic and favor singlet ground states. For concreteness, we consider the case where bonds 2 and 3 are Heisenberg, i.e., $D_{2}=D_{3}=0$. In this case the effective bond between spins 1 and 4 are $\tilde{J}_{14}=2 J_{3}\left(2 J_{1}-D_{1}\right) / 3 J_{2}$ and $\tilde{D}_{14}=0$. Therefore the generated bond is Heisenberg and ferromagnetic if

$$
J_{1}-D_{1} / 2<0 \text {. }
$$

Clearly, bond 1 can be AF and satisfy Eq. (6) if it lies in the shaded region of Fig. 1: The ground state of a bond in the lower part of the hatched region is a singlet, representing a genuine antiferromagnetic coupling; while in the upper part the ground state is a triplet, which also leads to a singlet ground state for the entire chain in the absence of randomness.

We have verified the above results of perturbation theory, that it is possible to get $S_{\text {tot }}=2$ ground states when Eq. (6) is satisfied, by performing exact diagonalization of

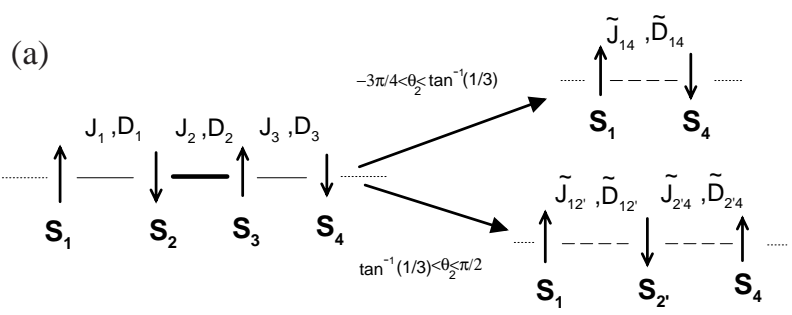

(b)

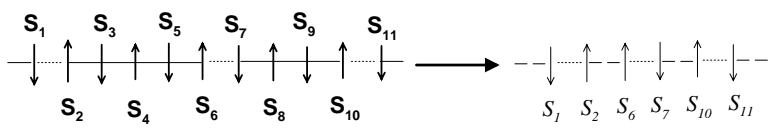

FIG. 2. Illustration of spin decimation procedures. (a) Strong randomness case. When the strongest bond (between spins 2 and 3 ) has a singlet ground state, spins 2 and 3 are decimated and an effective bond connecting 1 and 4 is generated; when the ground state is a triplet, 2 and 3 form an effective spin-1 object $S_{2^{\prime}}$, which is coupled to its neighbors 1 and 4. (b) Dilute randomness case. When a uniform spin-1 chain in the Haldane phase is broken into finite segments coupled by weak impurity bonds (dotted lines), the low energy degrees of freedom are half spins living at the edges of each segments; there is weak coupling between neighboring half spins, both in the same segment (broken lines) and different segments (dotted lines). 
four spin clusters. The results of one such calculation are shown in Fig. 3: as a function of $J_{2}$, the ground state of the cluster changes from $S_{\text {tot }}=0$ (which evolves from a product of singlets between spins $S_{1}$ and $S_{2}$ and $S_{3}$ and $S_{4}$ in the limit $J_{2}=0$ ) to $S_{\text {tot }}=2$.

The perturbative RG described above is reliable when the randomness is strong and the distributions of the couplings are broad $[1,10]$. We now consider the opposite limit of dilute randomness, namely a uniform AF spin1 chain in the Haldane phase $(-\pi / 4<\theta<\pi / 4$; see Fig. 1), with a finite Haldane gap $\Delta$ and a small fraction of impurity bonds that are much weaker than $\Delta$ [see Fig. 2(b)]. Here one must identify the true low energy degrees of freedom [8]. If the impurity bonds were taken away, the original chain would have been chopped into decoupled segments; the low-energy degrees of freedom are the two spin-1/2 at the two edges of each segment with a coupling (that can be either F or AF) decreasing exponentially with the length of the segment. Putting back the impurity bonds, as long as they are weak compared to $\Delta$, does not alter the bulk structure of the segments; their primary effect is to couple neighboring edge spins in different segments. Let us assume bond 1 coupling spins 1 and 2 is such an impurity bond, with $J_{1}, D_{1} \ll \Delta$. To calculate the coupling between the two edge spin-1/2s, which we label $1^{\prime}$ and $2^{\prime}$, we project the original operators onto the subspace of states below the Haldane gap, i.e., states of the effective edge spins [16]:

$$
\tilde{H}_{1^{\prime} 2^{\prime}}=P H_{12} P=J_{1} P \mathbf{S}_{1} \cdot \mathbf{S}_{2} P+D_{1} P\left(\mathbf{S}_{1} \cdot \mathbf{S}_{2}\right)^{2} P,
$$

where $P$ is the projection operator. Rotational symmetry as well as properties of spin- $1 / 2$ guarantee that $\tilde{H}_{1^{\prime} 2^{\prime}}=$ $\tilde{J}_{1^{\prime} 2^{\prime}} \mathbf{S}_{1^{\prime}} \cdot \mathbf{S}_{2^{\prime}}+C_{1^{\prime} 2^{\prime}}$, where $C_{1^{\prime} 2^{\prime}}$ is a constant. We also

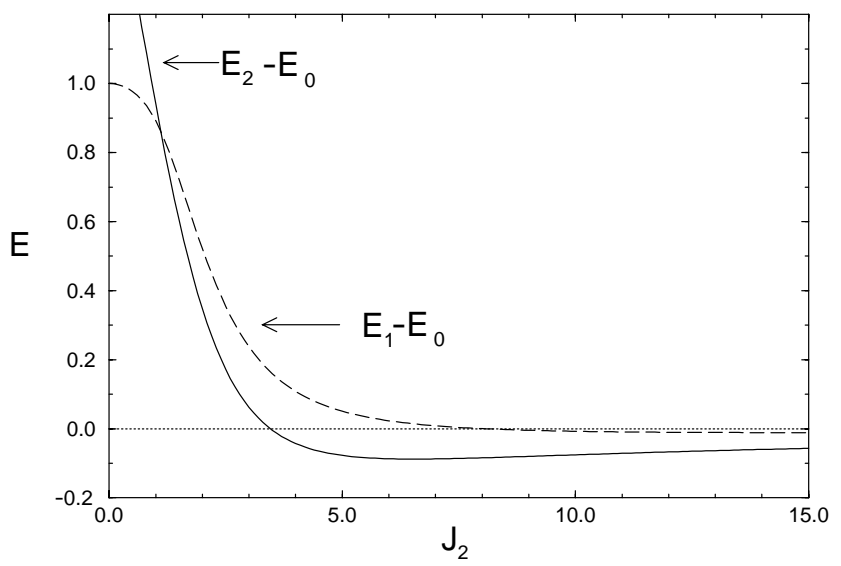

FIG. 3. Ground state level crossing in a four-spin cluster for $J_{1}=-1.0, D_{1}=-1.5, J_{3}=1.0, D_{2}=D_{3}=0$. The plot shows energies of the lowest energy quintuplet $\left(S_{\text {tot }}=2\right.$, solid line) and triplet ( $S_{\text {tot }}=1$, broken line) states, measured from lowest energy singlet state. For $J_{2}>3.46$, the ground state has total spin $S_{\text {tot }}=2$, despite the fact that all three bonds favor singlet ground states. have $P \mathbf{S}_{1} \cdot \mathbf{S}_{2} P=P \mathbf{S}_{1} P \cdot P \mathbf{S}_{2} P$, because spins 1 and 2 live in decoupled Hilbert spaces if bond 1 were not there, and the Wigner-Eckart theorem [17] guarantees $P \mathbf{S}_{i} P=$ $\alpha \mathbf{S}_{i}^{\prime}$. The constant $\alpha$ depends on bulk properties of the segments; for an infinitely long segment with Heisenberg coupling, $\alpha \approx 1.0640$ [18]. Similarly, $P\left(\mathbf{S}_{1} \cdot \mathbf{S}_{2}\right)^{2} P=$ $a+b \mathbf{S}_{1^{\prime}} \cdot \mathbf{S}_{2^{\prime}}$, and the important constant $b$ may be determined by calculating certain matrix elements of $\left(\mathbf{S}_{1}\right.$. $\left.\mathbf{S}_{2}\right)^{2}$ in the subspace: $b=2\left[\left\langle\uparrow_{1^{\prime}} \uparrow_{2^{\prime}}\left|\left(\mathbf{S}_{1} \cdot \mathbf{S}_{2}\right)^{2}\right| \uparrow_{1^{\prime}} \uparrow_{2^{\prime}}\right\rangle-\right.$ $\left.\left\langle\uparrow_{1^{\prime}} \downarrow_{2^{\prime}}\left|\left(\mathbf{S}_{1} \cdot \mathbf{S}_{2}\right)^{2}\right| \uparrow_{1^{\prime}} \downarrow_{2^{\prime}}\right\rangle\right]$. Using the commutation relations of $\mathbf{S}$ and the fact [18] $\left\langle\uparrow_{1^{\prime}}\left|S_{1}^{Z}\right| \uparrow_{1^{\prime}}\right\rangle=\alpha / 2$, etc., we obtain $b=-\alpha^{2} / 2$. Therefore $\tilde{J}_{1^{\prime} 2^{\prime}}=\alpha^{2}\left(J_{1}-D_{1} / 2\right)$, and again we find $\tilde{J}_{1^{\prime} 2^{\prime}}$ may be ferromagnetic even when the original bond 1 was $\mathrm{AF}$, with the same condition as before, namely Eq. (6). We have thus demonstrated the existence of such an instability to form ferromagnetic couplings in both the high disorder and dilute disorder limits.

We now discuss the origin of this ferromagnetic instability, and in particular, the significance of the special combination of $J$ and $D$ in Eq. (6). By introducing a new coupling constant $K=J-D / 2$, the RG equations (2) and (4) simplify significantly:

$$
\tilde{K}_{14}=\frac{4 K_{1} K_{3}}{3\left(K_{2}-5 D_{2} / 2\right)} ; \quad \tilde{K}_{12^{\prime}}=K_{1} / 2 .
$$

Combining these with Eqs. (3) and (5), we find $K$ and $D$ decouple except through energy denominators, and one cannot generate $K$ from $D$, or vice versa. This suggests that $K$ and $D$ represent couplings of operators with different symmetry properties.

To proceed further, we note that products of different components of the spin operator (that appear in the Hamiltonian) may be organized to form traceless irreducible spherical tensor operators [17]: $Y_{l m}(\mathbf{S})$, which is defined by replacing $\cos \theta$ by $S_{z}, \sin \theta \cos \phi$ by $S_{x}$, and $\sin \theta \sin \phi$ by $S_{y}$ in the usual spherical harmonics $Y_{l m}(\theta, \phi)$, and symmetrizing noncommuting components. $Y_{l m}(\mathbf{S})=0$ for $l>2 S$. A general way to write down the coupling between two spins that respect rotational symmetry [equivalent to $H_{i j}=\sum_{n=1}^{2 S} J^{(n)}\left(\mathbf{S}_{i} \cdot \mathbf{S}_{j}\right)^{n}$ ] is

$$
H_{i j}=\sum_{l=0}^{2 S} K^{(l)} \sum_{m=-l}^{l}(-1)^{m} Y_{l m}\left(\mathbf{S}_{i}\right) Y_{l,-m}\left(\mathbf{S}_{j}\right),
$$

where $K^{l}$ is the coupling constant of rank $l$ spherical tensors. It is easy to verify that

$$
\begin{aligned}
& \mathbf{S}_{1} \cdot \mathbf{S}_{2}=\frac{4 \pi}{3} \sum_{m=-1}^{1}(-1)^{m} Y_{1 m}\left(\mathbf{S}_{1}\right) Y_{1,-m}\left(\mathbf{S}_{2}\right) \\
&\left(\mathbf{S}_{1} \cdot \mathbf{S}_{2}\right)^{2}=-\frac{2 \pi}{3} \sum_{m=-1}^{1}(-1)^{m} Y_{1 m}\left(\mathbf{S}_{1}\right) Y_{1,-m}\left(\mathbf{S}_{2}\right) \\
&+\frac{8 \pi}{15} \sum_{m=-2}^{2}(-1)^{m} Y_{2 m}\left(\mathbf{S}_{1}\right) Y_{2,-m}\left(\mathbf{S}_{2}\right) \\
&+\frac{1}{3} \mathbf{S}_{1}^{2} \mathbf{S}_{2}^{2} .
\end{aligned}
$$


Therefore for the coupling of the form $J \mathbf{S}_{1} \cdot \mathbf{S}_{2}+D\left(\mathbf{S}_{1}\right.$. $\left.\mathbf{S}_{2}\right)^{2}$, we have $K^{(1)}=\frac{2 \pi}{3}(2 J-D) \propto K$, and $K^{(2)}=$ $\frac{8 \pi}{15} D$. Thus the $K$ variable is proportional to the coupling between rank 1 tensors (vectors), nothing but the Heisenberg coupling (in this tensor representation); and Eq. (6) indicates the Heisenberg coupling is ferromagnetic.

The advantage of writing the Hamiltonian in terms of couplings of irreducible spherical tensors instead of powers of $\mathbf{S}_{1} \cdot \mathbf{S}_{2}$ is that different symmetry properties of tensors with different ranks do not allow them to mix in first and second order perturbation calculations; while $\left(\mathbf{S}_{1} \cdot \mathbf{S}_{2}\right)^{n}$ in general includes tensor couplings with all ranks up to $n$. For example, we know $Y_{l m}(\mathbf{S})$ acting on a singlet creates an eigenstate of $\mathbf{S}_{\mathrm{tot}}^{2}$ and $S_{\text {tot }}^{z}$ with $S_{\text {tot }}=l$ and $S_{\text {tot }}^{z}=m$, therefore in the second order perturbation a coupling of rank $l$ between spins 1 and 4 is mediate through the channel of excited states with $S_{\text {tot }}=l$ of spins 2 and 3. More remarkably, when projecting spin1 couplings to couplings between spin-1/2 edge spins, the fact that spin- $1 / 2$ object does not support tensors with ranks higher than 1 guarantees that the coupling must be proportional to $J-D / 2$ of the original coupling, and the original rank 2 tensor coupling simply gets eliminated.

The above discussion also gives us insights into the origin of the ferromagnetic instability we demonstrated. Even though a single bond favors a singlet ground state, it may well contain ferromagnetic couplings between tensors with certain rank. In the absence of randomness no ferromagnetic instability is triggered by such couplings. In the presence of randomness, however, energy scales in the system get separated, and low- and high-energy subspaces of the Hilbert space get perturbatively decoupled (which is the basis for perturbative RG). In projecting to low-energy subspaces, certain AF couplings may get suppressed for symmetry reasons (as we have seen), while the original subdominant ferromagnetic couplings may survive and become dominant. In the example we illustrated above, the AF rank 2 couplings are suppressed in the low energy subspace, while the ferromagnetic rank 1 (Heisenberg) coupling survives. This is the origin of the ferromagnetic instability, which is a purely quantum mechanical effect, and absent without randomness.

With the generation of ferromagnetic bonds between segments as well as within segments, the decimated chain is very different from that obtained with pure Heisenberg coupling by Hyman and Yang [8]. In fact, it becomes of the universality class of the spin- $1 / 2$ chain with random $\mathrm{AF}$ and $\mathrm{F}$ couplings studied by Westerberg et al. [11]. Using our formalism, their RG scheme may be easily generalized to include higher spins and non-Heisenberg couplings, as flows of couplings of different rank tensors tend to decouple. Our results show that at low energies high rank couplings are strongly suppressed, if the original couplings are dominantly Heisenberg, which is a likely situation in nature [19]. Therefore the active degrees of freedom in our case will be weakly coupled large moments as in the case of Westerberg et al., which lead to a pure Curie susceptibility in the low temperature limit.

In summary, we have demonstrated the possibility of generating ferromagnetic couplings in quantum spin chains with random antiferromagnetic couplings. Though we have discussed $S=1$ exclusively for concreteness, similar effects would be expected for higher spins. However, as our treatment shows, the effects are purely quantum mechanical, and dependent on either the spin gap or the region of validity of second order perturbation theory, both of which are known to become smaller with increasing $S$. Consequently, we expect the region of this anomalous behavior to decrease with increasing $S$, and disappear in the classical limit $S \rightarrow \infty$.

This work was supported by NSF Grant No. DMR9400362 (at Princeton), as well as support from Caltech, and the Aspen Center for Physics.

[1] S. K. Ma et al., Phys. Rev. Lett. 43, 1434 (1979); C. Dasgupta and S. K. Ma, Phys. Rev. B 22, 1305 (1980).

[2] R. N. Bhatt and P. A. Lee, Bull. Am. Phys. Soc. 25, 206 (1980); Phys. Rev. Lett. 48, 344 (1982).

[3] R. N. Bhatt, Physica (Amsterdam) 109B \& 110B, 2145 (1982).

[4] F. D. M. Haldane, Phys. Lett. 93A, 464 (1983).

[5] D. S. Fisher, Phys. Rev. B 51, 6411 (1995).

[6] H. Rieger and A. P. Young, Phys. Rev. B 54, 3328 (1996); M. Guo, R. N. Bhatt, and D. A. Huse, ibid. 54, 3336 (1996).

[7] R. A. Hyman et al., Phys. Rev. Lett. 76, 839 (1996); Kun Yang et al., J. Appl. Phys. 79, 5096 (1996).

[8] R. A. Hyman and Kun Yang, Phys. Rev. Lett. 78, 1783 (1997); R. A. Hyman, Ph.D. thesis, Indiana University, 1996.

[9] C. Monthus et al., Phys. Rev. Lett. 79, 3254 (1997).

[10] D. S. Fisher, Phys. Rev. B 50, 3799 (1994).

[11] E. Westerberg et al., Phys. Rev. Lett. 75, 4032 (1995); Phys. Rev. B 55, 12578 (1997). See also B. Fischmuth and M. Sigrist, Phys. Rev. Lett. 79, 147 (1997); K. Hida, J. Phys. Soc. Jpn. 66, 330 (1997).

[12] B. Boechat et al. [Solid State Commun. 98, 411 (1996)] studied a spin-1 chain with biquadratic couplings only.

[13] A. Klumper, Europhys. Lett. 9, 815 (1989); M. T. Batchelor and M. N. Barber, Phys. Rev. B 40, 4621 (1989).

[14] G. Fath and J. Soloym, Phys. Rev. B 44, 11836 (1991); C. Itoi and M.-H. Kato, Phys. Rev. B 55, 8295 (1997).

[15] The following two equations agree with those of Hyman in Ref. [8], and reduce to those in Ref. [12] in certain limiting cases only.

[16] E.S. Sorensen and I. Affleck, Phys. Rev. B 51, 16115 (1995).

[17] See, e.g., J.J. Sakurai, Modern Quantum Mechanics (Addison-Wesley, New York, 1985).

[18] S. R. White, Phys. Rev. Lett. 69, 2863 (1992); E. S. Sorensen and I. Affleck, Phys. Rev. B 49, 15771 (1994).

[19] R. Bhatt and K. Yang, J. Appl. Phys. (to be published). 